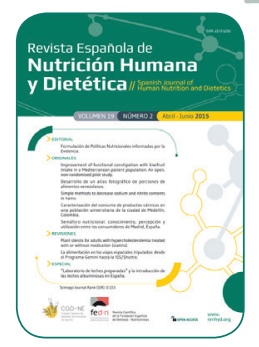

\title{
REVISIÓN
}

\section{La alimentación en los viajes espaciales tripulados: desde el Programa Gemini hasta la ISS/Shuttle}

\author{
Juan Antonio Gomar-Serrano ${ }^{\mathrm{a}}$, José Miguel Soriano-Del Castillo ${ }^{\mathrm{a}}$, Laura Bilbao-Cercós ${ }^{\mathrm{b}}$, \\ a Departamento de Medicina Preventiva, Salud Pública, Ciencias de la Alimentación, Toxicología y Medicina Legal, Facultad de \\ Farmacia, Universidad de Valencia, España \\ b Clínica Universitaria de Nutrición, Actividad Física y Fisioterapia (CUNAFF VLC/CAMPUS), España. \\ *laura.bilbao@uv.es
}

Recibido el 29 de julio de 2014; aceptado el 9 de diciembre de 2014.

La alimentación en los viajes espaciales tripulados: desde el Programa Gemini hasta la ISS/Shuttle

\section{PALABRAS CLAVE RESUMEN}

Viaje espacial;

Los alimentos espaciales han tenido una gran importancia en el desarrollo de las misiones espaciales tripuladas ya que constituyen la única fuente de energía y nutrientes para el ser

Astronautas;

Alimentos;

Envasado de alimentos. humano fuera del planeta Tierra. Dichos alimentos han experimentado una gran evolución desde el inicio del Programa Mercury hasta las actuales misiones a la International Space Station (ISS). En un primer momento eran diseñados para ofrecer una gran densidad energética y nutritiva y actualmente se da prioridad a las características organolépticas y psicológicas, ya que contribuyen a un mayor consumo y un mejor estado nutricional de los astronautas. EI objetivo del presente estudio es hacer un análisis retrospectivo de las publicaciones hasta la fecha sobre alimentos espaciales para ver si los disponibles actualmente son apropiados para mantener un buen estado nutricional de los astronautas en el marco de una misión espacial tripulada al espacio profundo. Para la búsqueda de estos artículos se ha utilizado la base de datos de PubMed y de la web del Jet Propulsion Laboratory de la National Aeronautics and Space Administration (NASA), de los artículos publicados hasta el año 2012. 
Food in manned spaceflight: from Gemini Program to the ISS/Shuttle programs

\section{KEYWORDS}

Space flight;

Astronauts;

Food;

Food packaging.

\section{ABSTRACT}

Space foods have had great importance in the development of manned space missions since they constitute the only source of energy and nutrients for the human being outside the planet Earth. These foods have undergone a great evolution since the beginning of the Mercury Program to current missions to the International Space Station (ISS). Initially they were designed to provide a great energy and nutrient density and currently priority is given to psychological and organoleptic characteristics since they contribute to increased consumption and a better nutritional status of astronauts. The aim of the present study is to make a retrospective analysis of what has been published until today about the space food, since in the context of a possible future mission manned to deep space, available food are not currently suitable for ensuring an optimal state of health of the astronauts. To search for these items has been used database PubMed and web of the jet propulsion laboratory (Jet propulsion laboratory) National Aeronautics and Space Administration NASA articles published up to 2012.

\section{CITA}

Gomar-Serrano JA, Soriano-Del Castillo JM, Bilbao-Cercós L. La alimentación en los viajes espaciales tripulados: desde el Programa Gemini hasta la ISS/Shuttle. Rev Esp Nutr Hum Diet. 2015; 19(2): 116 - 123. D0I: 10.14306/ renhyd.0.0.116

\section{INTRODUCCIÓN}

Un alimento espacial es un tipo especial de alimento empleado para la nutrición de los astronautas en las misiones tripuladas al espacio. Fundamentalmente se trata de alimentos básicos que cubren nutritivamente las necesidades medias de los astronautas en el espacio. Dentro de los alimentos espaciales se debe diferenciar entre los pre-envasados y los alimentos frescos producidos en el espacio mediante los sistemas de biorregeneración de alimentos ${ }^{1-12}$. Los primeros son producidos, procesados y envasados en nuestro planeta y se proporcionan a los astronautas listos para consumir o prácticamente listos. Los segundos, en cambio, son alimentos que se producen básicamente en un invernadero donde se recrearían todas las condiciones ambientales necesarias para el crecimiento de los cultivos.

Actualmente, la información publicada en España sobre la nutrición en los viajes espaciales es muy escasa, por lo que realizar una revisión bibliográfica de la información publicada hasta el momento por otros países para realizar una puesta al día del tema y hacer una publicación en la lengua española resulta de gran interés. El principal motivo es que a día de hoy se está planeando la posibilidad de realizar misiones espaciales tripuladas a lugares mucho más lejanos que los que se ha viajado hasta ahora y no se debe olvidar que la correcta nutrición de las personas que serán enviadas en dichas misiones resulta de vital importancia para garantizar el éxito de las misiones, la supervivencia y el buen estado de salud de los astronautas. La alimentación de las personas que viajan al espacio es un factor que puede condicionar el desarrollo de futuras misiones espaciales al espacio profundo. Es de vital importancia conocer las características de los alimentos espaciales disponibles hoy en día y si serían capaces de sustentar la vida de los seres humanos que se encontrarían fuera del planeta tierra o si por el contrario es necesario un mayor desarrollo tecnológico. Asimismo, se debe contemplar la posibilidad de que parte de los alimentos usados en una futura misión espacial pudieran ser producidos fuera de nuestro planeta y la tecnología necesaria para ello. 
Así, este trabajo pretende realizar una revisión bibliográfica de la nutrición en los viajes espaciales tripulados, centrándose exclusivamente en los alimentos pre-envasados, con el objetivo de realizar una puesta al día del tema, además de conocer los tipos y sus características, cuál ha sido su evolución a lo largo del tiempo y conocer si se cubren los requerimientos de los astronautas.

\section{Evolución de los alimentos suministrados en las misiones espaciales desde el Programa Gemini hasta la ISS/Shuttle}

El sistema de suministro de comida de la NASA empezó con productos especiales que eran diseñados para tener el menor volumen y tamaño posible debido a las limitaciones de masa y volumen que podían ser lanzados fuera de la Tierra. Sin embargo fue evolucionando progresivamente hacia alimentos con mejor palatabilidad debido a las recomendaciones de los médicos, ya que favorece un mejor estado nutricional ${ }^{13}$.

En el Programa Gemini de la NASA el suministro de alimentos se basaba en cubitos pequeños de carne, fruta, postres y pan (Figura 1.1) que fueron diseñados para ofrecer $2.890 \mathrm{Kcal}$ en $730 \mathrm{~g}$ de alimento envasado ${ }^{14}$. La aceptabilidad de estos cubitos por la tripulación no fue demasiado buena y muchos eran devueltos a la Tierra sin ser $\operatorname{comidos}^{13}$.

En el inicio del Programa Apollo se mantuvieron muchos de los alimentos usados en el Programa Gemini, sin embargo fue evolucionando paulatinamente mientras se desarrollaba la tecnología que permitiría incluir una mayor variedad de alimentos para promover un mayor consumo por parte de los astronautas $^{15}$. En el Apollo 7 se introdujo mayor cantidad de alimentos rehidratables (Figura 1.2) que incrementaron la masa hasta $820 \mathrm{~g}$ de comida envasada por persona y día ${ }^{16}$. En el Apollo 8 la tripulación prefería los nuevos alimentos termoestabilizados y en el Apollo 14 el peso de la comida por astronauta y día ascendió a 1.100g. El aumento de peso estaba justificado por las preferencias de los astronautas respecto a la comida ${ }^{13}$.

En el Skylab de la NASA se incrementó la palatabilidad y la variedad de los menús respecto al Programa Apollo. Los habitantes del laboratorio disponían de 72 alimentos entre los que elegir. Todos los alimentos necesarios se lanzaron en la primera misión con lo que tendrían más de dos años cuando la última misión del programa se llevara a cabo, por lo que muchos alimentos se envasaban en latas de aluminio para

Figuras 1.1, 1.2, 1.3 y 1.4. Alimentos usados pr la NASA en las misiones espaciales tripuladas.

1

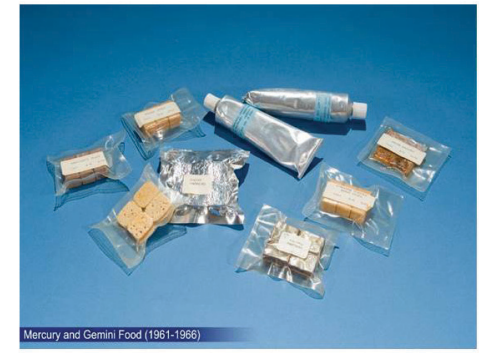

3

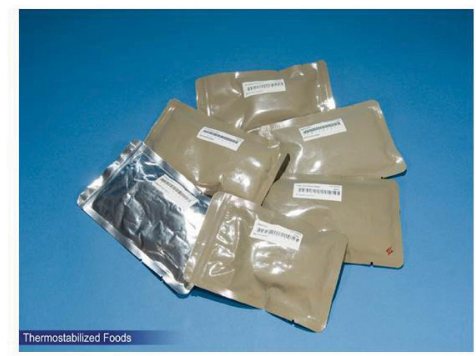

2

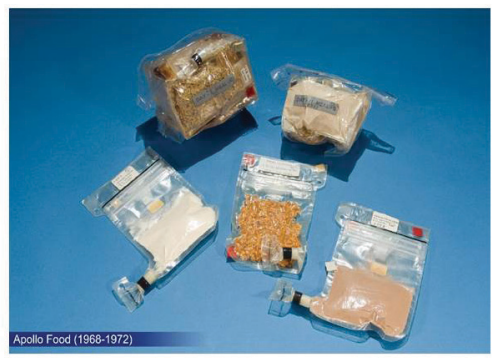

4

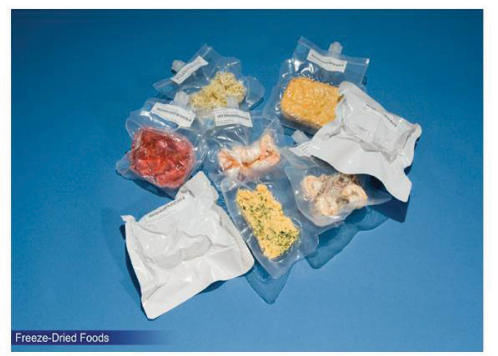

Figura 1.1: Alimentos usados en los Programas Mercury y Gemini. Figura 1.2: Alimentos usados en el Programa Apollo.
Figura 1.3: Ejemplos de alimentos termoesbilizados. Figura 1.4: Ejemplos de alimentos liofilizados de la NASA. 
extender su vida útil más allá de dos años ${ }^{13}$. Fue el primer programa espacial en incluir congeladores, refrigeradores y equipos para el calentado de los alimentos ${ }^{15}$.

Los miembros de las misiones de la ISS y del transbordador (Shuttle) recibían aproximadamente $1.800 \mathrm{~g}$ de comida por persona y día, incluyendo mayor proporción de alimentos termoestabilizados (Figura 1.3) respecto a las misiones ApoIlo, debido a que eran preferidos por los astronautas y los alimentos liofilizados (Figura 1.4) son menos aceptados. Los motores del transbordador espacial usaban hidrógeno como combustible con lo que se producía agua durante su funcionamiento. Ésta era usada para rehidratar los alimentos deshidratados y liofilizados, por lo que el $50 \%$ de los alimentos del transbordador eran lanzados en forma deshidratada ${ }^{13}$. La utilización de alimentos liofilizados o deshidratados en la ISS no aporta ventajas significativas de ahorro de masa, debido a que utiliza paneles solares para producir energía y no otros sistemas que generan agua como subproducto, por tanto se requiere el envío de agua desde la Tierra para rehidratarlos ${ }^{13}$. El programa del transbordador espacial fue cancelado por la NASA en el año 2011.

\section{Importancia de los alimentos ricos en nutrientes y energía}

La NASA ha estudiado diversas estrategias para reducir la masa de la comida necesaria para alimentar a los astronautas manteniendo una dieta adecuada. Una forma de reducir la masa de la comida envasada fue posible mediante una mayor variedad de los menús ${ }^{13}$. Uno de los objetivos fue mantener el número de calorías suministradas a la población pero aumentando la densidad nutritiva y energética de los alimentos, maximizando el porcentaje de energía aportado por las grasas hasta el máximo recomendado (35\%). De esta manera, se redujeron las necesidades de hidratos de carbono y proteína, lo que contribuyó a disminuir la masa total de la comida. Sin embargo, el aumento del contenido graso de los alimentos puede afectar negativamente a su tiempo de vida útili13. Adicionalmente, algunas pruebas sobre la aceptabilidad de los alimentos por los astronautas han mostrado que los niveles de humedad de los alimentos termoestabilizados puede ser reducida en un $10 \%$. Reduciendo la humedad de estos alimentos hasta ese nivel e incrementando la energía aportada por las grasas hasta el $35 \%$ se pudo reducir el peso de los alimentos necesarios en $321 \mathrm{~g}$ por astronauta y día. Una segunda parte del estudio de la reducción de la masa de los alimentos necesarios mostró cómo la sustitución de una de las comidas del día por un sustituto de comida resulta en una reducción de $240 \mathrm{~g}^{13}$. Por tanto, si se combina la sustitución de una comida diaria por un sustituto de comida con la reducción de la humedad de los alimentos termoestabilizados y el aumento de aporte de grasa se podría reducir la masa de alimentos necesarios en 561g por astronauta y día, lo que equivaldría a una disminución del $36 \%$ del peso total de la comida necesaria ${ }^{13}$.

Por otro lado, la NASA estudió diferentes alimentos para conocer la relación entre la densidad energética y nutritiva con la masa, con el fin de conocer qué alimentos serían la mejor fuente de nutrientes y energía para sus astronautas. El trabajo de Stoklosa (2009) ${ }^{17}$ determinó la eficiencia del sistema actual de aprovisionamiento de comida a los astronautas basándose en la relación entre la masa de los alimentos y su riqueza nutritiva. Este estudio se basó en el Naturally Nutrient Rich score que mide la densidad en nutrientes y calorías de un grupo de alimentos seleccionados, entre los cuales se incluyen bebidas, vegetales, sopas, huevos, frutas, entrantes, cereales y panes, postres, legumbres y nueces. Los nutrientes elegidos para el estudio fueron: proteína, calcio, hierro, vitamina $A$, vitamina $C$, tiamina, riboflavina, vitamina $B_{12^{\prime}}$ folato, vitamina $D$, vitamina $E$, magnesio, potasio, zinc, fibra y ácido pantoténico ${ }^{13,17}$.

De todos los alimentos estudiados, los que presentaron mayor cantidad de nutrientes son las bebidas, seguidas por los vegetales y las sopas. A pesar de esta riqueza en nutrientes el aporte calórico de estos alimentos es muy bajo, con lo que basar la alimentación en ellos requeriría el lanzamiento de enormes cantidades de masa desde la Tierra. Dada la importancia de cubrir tanto las necesidades de energía como de nutrientes de la tripulación en los viajes espaciales de larga duración, se deben seleccionar aquellos alimentos que ofrezcan el mejor equilibrio entre aporte de nutrientes y densidad calórica lo que permitiría reducir la masa de comida a ser lanzada al espacio. De todos los alimentos seleccionados, las nueces fueron el alimento más equilibrado en cuanto a estas dos variables ya que contienen una significativa cantidad de nutrientes y una gran densidad calórica ${ }^{13}$

Aportando alimentos que contengan gran densidad de nutrientes y calorías se consigue una reducción de la masa que debe ser lanzada para suministrar alimentos a los astronautas, con lo que se reduce la cantidad de propelente necesario para llevar la nave espacial fuera de la Tierra. La reducción de la masa puede ser lograda reformulando los productos y aportando barritas de comida. El mayor reto en la reformulación de los alimentos es mantener su estabilidad durante el almacenamiento prolongado y que no se vean perjudicadas sus características organolépticas y la aceptabilidad de estos por parte de la tripulación ${ }^{13}$.

\section{Importancia del envasado de los alimentos}

El envasado de los alimentos fue el mayor contribuyente al volumen y masa de la basura generada en las misiones de la NASA, ya que aproximadamente el $12,5 \%$ del peso de los 
alimentos rehidratables usados en el transbordador se convertía en basura ${ }^{13}$. Sin embargo, no se puede prescindir del envasado ya que es necesario para mantener la seguridad, la calidad nutricional y la aceptabilidad de los alimentos: su función es protegerlos de las agresiones del ambiente exterior causadas por microorganismos, radiación, humedad, oxígeno y otros. Es de vital importancia que el envasado de los alimentos los proteja del oxígeno del ambiente ya que el contacto con el oxígeno produce oxidación y pérdidas nutricionales. También debe ofrecer protección frente a la humedad ya que puede alterar la calidad de rehidratación $n^{13,15}$.

En los envasados actuales se usan dos capas para proteger los alimentos. La primera capa usada para alimentos liofilizados y en forma natural no ofrece una buena protección frente al oxígeno y la humedad para mantener estos alimentos en buenas condiciones durante 18 meses, sin embargo sí favorece una correcta inspección visual de los alimentos para comprobar su estado. La segunda capa, que contiene aluminio, se usa para proporcionar la protección necesaria frente al oxígeno y la humedad. La incineración de la basura ha sido propuesta como solución a la acumulación de residuos en futuras misiones. El uso de papel de aluminio complica la futura incineración de la basura ya que no se quema completamente y deja cenizas ${ }^{18}$. Además, otro inconveniente del uso de papel de aluminio es que no permite la inspección ocular de los productos una vez han sido envasados ${ }^{12}$.

En el año 2009, los investigadores del proyecto Advanced Food Technology ${ }^{19}$ evaluaron la posibilidad de abandonar la práctica de usar una segunda capa de protección en el envasado. Así, en lugar de usar dos capas, se propuso el uso de una sola capa más grande para preservar los alimentos reduciendo en un $20 \%$ el volumen y en un $50 \%$ el tiempo necesario para el envasado ${ }^{13,19}$. Esta práctica permitiría reducir los residuos generados en una futura misión espacial. Otra forma de reducir los residuos sería el uso de materiales alternativos para el envasado como el uso de una sola capa clara cubierta de óxido de aluminio contra el actual sistema de envasado. Se ha visto que es apropiado para las expediciones a la ISS por lo que se podría usar una sola capa para envasar los alimentos que son enviados a la ISS reduciendo el peso, sin embargo no es apto para una misión a Marte, ya que la vida útil de los alimentos no es suficientemente larga y no garantizarían un óptimo estado de salud de los astronautas en misiones de larga duración ${ }^{15}$.

Es de vital importancia, por tanto, encontrar nuevos materiales para el envasado de los alimentos que sean menos pesados, presenten una mayor protección de los alimentos para permitir su conservación durante más tiempo y sean compatibles con procesos de incineración, sin olvidar la importancia de la inspección visual de los alimentos para comprobar su correcto estado ${ }^{13}$.

\section{Vida útil y calidad de los alimentos usados en las misiones espaciales}

Los alimentos usados por la NASA en sus misiones tripuladas tenían una vida media entre 18 y 24 meses, lo que es suficiente para asegurar la alimentación adecuada de sus astronautas en las misiones actuales a la ISS. Sin embargo este tiempo sería insuficiente para misiones tripuladas de mayor duración, más allá de la ISS, como podría ser una futura misión tripulada a Marte ${ }^{15,20}$.

La calidad de los alimentos tuvo un gran impacto psicológico en los astronautas cuando se hallaban en una misión tripulada de larga duración ${ }^{13,15}$, más allá de la seguridad para la salud física de su consumo. Los alimentos usados para las misiones tripuladas se evaluaron usando métodos sensoriales para su aceptabilidad mediante una prueba con 30 consumidores. En el análisis se consideraron varias características de los alimentos como su sabor, textura y aroma, y según estas características se clasificaron en una escala de 9 puntos. En esta evaluación intervinieron diversos factores como la edad del consumidor, las condiciones de almacenamiento y el ambiente.

Un estudio realizado por el Johnson Space Center entre 2003 y 2008 $21-23$ mostró los cambios de calidad de los alimentos termoestabilizados. El estudio empezó con 13 alimentos termoestabilizados almacenados a 4,4 으 (control), $22^{\circ} \mathrm{C}$ (temperatura de almacenamiento de las misiones actuales) y 35 C (temperatura aumentada) y una humedad relativa del $50 \%$. El estudio terminaba en el momento en que el alimento se vuelve inaceptable o transcurridos 3 años. Se eligieron productos que son considerados representativos de los alimentos termoestabilizados usados actualmente ${ }^{13,21-24}$. Las carnes fueron los alimentos que se mantenían más estables en el tiempo, más de 3 años sin refrigeración. En segundo lugar se situaron las frutas y los postres que se mantenían entre 1,5 y 5 años sin refrigeración. Las féculas y los vegetales se mantenían estables entre 1,5 y 5 años sin refrigeración. Los huevos eran rechazados inmediatamente después de ser termoestabilizados. También se analizaron pérdidas nutricionales observándose pérdidas superiores al $40 \%$ en vitamina $C$, ácido fólico y ácido pantoténico en muchos de los productos, otros nutrientes no se vieron prácticamente alterados.

Estos resultados mostraron la necesidad de un nuevo procesado tecnológico y de mejorar las condiciones de almacenamiento para mantener la calidad inicial de los alimentos. Algunos análisis de la NASA mostraron que la vida media de productos secos puede ser mejorada reduciendo la cantidad de oxígeno en el alimento envasado al vacío, por lo que es necesario mejorar las técnicas de envasado para disminuir la cantidad de oxígeno que queda dentro de los productos envasados al vacío ${ }^{13}$. 
Otra alternativa para aumentar la vida útil de los alimentos podría ser utilizar el ambiente del espacio como un congelador externo a la nave ya que se estima que las bajas temperaturas son capaces de frenar la degradación de los alimentos. Sin embargo no se sabe cómo la radiación del espacio profundo podría afectar a los alimentos conservados por este método ya que no se ha estudiado ${ }^{13}$.

Actualmente 2 nuevas tecnologías están siendo evaluadas para conseguir la esterilidad comercial provocando menor daño a la matriz de los alimentos, lo que contribuiría a una mayor calidad del alimento procesado y a mejorar su conservación en el tiempo. Se trata del procesamiento a alta presión (HPP) y la esterilización con microondas. HPP utiliza presiones que llegan a las 6.000 atmosferas aproximadamente, acompañada o no de calor, con lo que se inactivan los microorganismos, se mantiene la frescura natural y se retiene la calidad de los alimentos aumentando la vida útil media. La esterilización con radiación por microondas utiliza altas temperaturas, 129 ㅇ $\mathrm{C}$ y tiempos cortos de 10 minutos, con ello se consigue una mayor calidad en los alimentos que con las técnicas actuales para la termoestabilización ${ }^{13}$.

A pesar de estos prometedores resultados se necesita más investigación para ver cómo afecta el almacenamiento prolongado a los alimentos procesados con estas innovadoras técnicas y si se mantiene el mayor valor nutricional inicial respecto a las técnicas usadas actualmente ${ }^{13}$.

Por otro lado, una forma de aumentar la aceptabilidad de los alimentos es aumentar la variedad del menú ${ }^{15}$ y que se puedan dar diversos usos a los ingredientes ya que cuando los alimentos se repiten cada 8 días en vez de cada 16, la aceptabilidad se ve reducida ${ }^{13}$. También el contexto social puede afectar a la aceptabilidad de los alimentos: promoviendo las comidas en grupo se reduce el estrés y el aburrimiento en las misiones prolongadas, pudiendo contribuir a una mayor aceptabilidad de los alimentos ${ }^{15}$.

\section{Importancia de los nutrientes y sus pérdidas en los viajes espaciales}

Los nutrientes son vitales para todos los procesos celulares en el cuerpo, tanto en la Tierra como en el espacio. El estado de ciertas vitaminas en el cuerpo se ve alterado durante misiones de larga duración ${ }^{25}$ pero no se sabe si estos cambios son debidos a alteraciones del metabolismo de las vitaminas o si las vitaminas presentes en los alimentos han sido degradadas debido almacenamiento durante largos periodos de tiempo u otros efectos, como las radiaciones ionizantes presentes en el espacio. Aunque esto último no ha sido estudiado, las radiaciones del espacio podrían degradar los nutrientes en los alimentos, disminuir su biodisponibilidad o crear sabores desagradables debido a procesos de oxidación.

El peso molecular, y por tanto el tamaño de las vitaminas, es mucho más pequeño que la molécula de ADN, por lo que la radiación encontrada típicamente en misiones espaciales tripuladas tiene menos probabilidades de afectar directamente a estas moléculas más pequeñas. Sin embargo, cuando las vitaminas están rodeadas de agua y lípidos, como lo están en los alimentos, la radiación produce radicales reactivos que pueden incrementar drásticamente la probabilidad de interacción con vitaminas susceptibles, especialmente las antioxidantes. Por tanto, se piensa que la exposición a la radiación y el almacenamiento durante largos periodos de tiempo asociado a misiones espaciales de larga duración deberían degradar las vitaminas altamente susceptibles.

En este sentido, Zwart y Cols. ${ }^{26}$ realizaron un estudio con el fin de ver la degradación que sufren los alimentos a lo largo del tiempo en la ISS comparándolos con un control en la Tierra. La elección de alimentos estuvo limitada por el volumen y la masa que pudiera ser lanzada en una nave espacial, y se basó en los alimentos usados por el sistema de suministros de la ISS, teniendo en cuenta que fueran buenas fuentes de alguna vitamina liposoluble durante al menos un año y que representaran los diferentes tipos de comida espacial, rehidratable y forma natural ${ }^{27}$. Se observó que el tiempo de almacenamiento tuvo un efecto mucho mayor en la pérdida de nutrientes que el hecho de haber pasado un tiempo fuera de la Tierra26.

La exposición de estos alimentos a la radiación presente en el espacio puede causar pérdidas de nutrientes y alteraciones de los alimentos, que se sumarían a las causadas por el paso del tiempo, sin embargo la dosis de radiación a la que se ven expuestos los alimentos en la ISS es baja y no se pueden extrapolar los resultados a lo que pasaría en una misión al espacio profundo donde la dosis de radiación que recibirían sería mucho mayor ${ }^{26}$.

El estudio de Zwart y Cols. ${ }^{26}$ sólo abarcó 880 días de duración, el equivalente a aproximadamente 2 años y medio, por lo que es de esperar que en periodos de tiempo más prolongados se produzcan pérdidas mayores de nutrientes, lo que podría poner en peligro la salud de los astronautas. Es necesario, por tanto, mejorar las técnicas de procesado de los alimentos que se suministraran desde la Tierra, así como el material usado para su envasado que permita una mejor conservación.

Además, durante las misiones tripuladas de cortos periodos de tiempo se han observado alteraciones en el estado nutricional de los astronautas ${ }^{25}$ que pueden ser explicadas, al menos parcialmente, por la alteración descrita de los 
nutrientes. Sin embargo, podrían producirse alteraciones metabólicas significativas durante los viajes espaciales de larga duración. Para continuar con la exploración espacial de un modo seguro, estas alteraciones nutricionales observadas en los astronautas deben ser mejor comprendidas.

\section{CONCLUSIONES}

Los alimentos espaciales han experimentado una notable evolución desde el comienzo de la exploración espacial tripulada hasta la actualidad, satisfaciendo, no sólo las necesidades energéticas y nutricionales, sino también las necesidades psicológicas, pasando a ser cada vez más importantes las características organolépticas. No obstante, aún no se dispone de alimentos capaces de asegurar un óptimo estado de salud en viajes espaciales al espacio profundo. Es fundamental que la densidad calórica y nutritiva de los alimentos seleccionados sea lo más alta posible para reducir la masa en la nave espacial y que su envasado sea el adecuado para reducir los residuos generados. Además, para maximizar la vida útil de los alimentos durante periodos de tiempo muy prolongados, es necesario estudiar cómo se ven alteradas las propiedades nutricionales de los alimentos preenvasados durante su almacenamiento fuera de la Tierra.

\section{conflicto de intereses}

Los autores expresan que no hay conflicto de intereses al redactar el manuscrito.

\section{BIBLIOGRAFÍA}

1. Zasypkin DV, Lee TC. Food processing on a space station: feasibility and opportunities. Life Support Biosph Sci. 1999; 6(1): 39-52

2. Schwartzkopf SH. Human life support for advanced space exploration. Adv Space Biol Med. 1997; 6: 231-53.

3. Zimmerman B. Heat transfer and cooking. Cooking for Engineers [revista en internet]. 2007 [consulta: 27/07/2014] Disponible en: http://www.cookingforengineers.com/ article/224/Heat-Transfer-and-Cooking

4. Gandolph J, El-Abiad M, Mauer L, Perchonok MH. Equivalent system mass (ESM) estimates for commercially available, small-scale food processing equipment. SAE Technical Paper. 2004; 01: 2526.

5. Gregson CM, Lee T-C. Considerations in the development of food processing and products for long-term space missions in a near self-sufficient environment. Spec Publ- R Soc Chem. 2001; 274: 477-91.

6. Fu B, Nelson PE. Conditions and constraints of food processing in space. Food Technol. 1994; 48(9): 113-22, 127, 204.

7. Rickman JC, Bruhn CM, Barrett DM. Nutritional comparison of fresh, frozen, and canned fruits and vegetables II. Vitamins $A$ and carotenoids, vitamin E, minerals and fiber. J Sci Food Agric. 2007; 87: 1185-96.

8. Rickman JC, Barrett DM, Bruhn CM. Nutritional comparison of fresh, frozen, and canned fruits and vegetables. Part 1. Vitamins C and B and phenolic compounds. J Sci Food Agric. 2007; 87: 930-44.

9. Kalt $W$. Effects of production and processing factors on major fruit and vegetable antioxidants. J Food Sci. 2005; 70(1): 1119.

10. Rock CL, Lovalvo JL, Emenhiser C, Ruffin MT, Flatt SW, Schwartz S]. Bioavailability of beta-carotene is lower in raw than in processed carrots and spinach in women. J Nutr. 1998; 128(5): 913-6.

11. Van het Hof KH, Tijburg LBM, Pietrzik K, Weststrate JA. Influence of feeding different vegetables on plasma levels of carotenoids, folate and vitamin C. Effect of disruption of the vegetable matrix. J Nutr. 1999; 82(3): 203-12.

12. Dewanto $\mathrm{V}, \mathrm{Wu} \mathrm{X}$, Adom KK, Liu RH. Thermal processing enhances the nutritional value of tomatoes by increasing total antioxidant activity. J Agric Food Chem. 2002; 50(10): 3010-4.

13. Cooper M, Douglas G, Perchonok M. Developing the NASA food system for long-duration missions. J Food Sci. 2011; 76(2): R40-8.

14. Huber CS, Heidelbaugh ND, Smith MC, Klicka M. Space foods. En: Birch GG, Green LF, Plaskett LG, editores. Health and foods. New York: John Wiley and Sons; 1972. p. 130-51.

15. Perchonok MH, Cooper MR, Catauro PM. Mission to Mars: food production and processing for the final frontier. Annu Rev Food Sci Technol. 2012; 3: 311-30.

16. Smith MC, Heidelbaugh ND, Rambaut PC, Rapp RM, Wheeler $\mathrm{HO}$, Huber CS, et al. Apollo food technology. En: Johnston RS, Dietlein LF, Berry CA. Biomedical results of Apollo. Washington: Scientific and Technical Information Office, National Aeronautics and Space Administration, U.S. Govt. Print. Off.; 1975. p. 437-84.

17. Stoklosa A. Packaged food mass reduction trade study. NASA Advanced Capabilities Division Research \& Technology Task Book [revista en internet]. 2009 [consulta: 27/07/2014]. Disponible en: http://taskbook.nasaprs.com/ Publication/index.cfm?action=public_query_taskbook content\&TASKID=7369

18. Wydeven T, Golub MA. Waste streams in a crewed space habitat. Waste Manag Res. 1991; 9: 91-101.

19. Oziomek T, Catauro P. Bulk overwrap packaging. NASA Advanced Capabilities Division Research \& Technology Task Book [revista en internet]. 2009 [consulta: 27/07/2014]. Disponible en: http://taskbook.nasaprs.com/ Publication/index.cfm?action=public_query_taskbook content\&TASKID=7595

20. Hoffman S], Kaplan DI, Mars Exploration Study Team. Human exploration of Mars: the reference mission of the NASA Mars Exploration Study Team. Houston, Tex.: National Aeronautics and Space Administration, Lyndon B. Johnson Space Center; 1997.

21. Perchonok MH, Swango B, Stevens I, Clynch M. Shelf Life Determination of Thermally Processed Foods. SAE International [revista en internet]. 2003 [consulta: 27/07/2014]; 2003-012621. Disponible en: http://papers.sae.org/2003-01-2621/

22. Perchonok M. Thermostabilized food study (former title-shelf life determination of thermally processed 
foods). NASA Advanced Capabilities Division Research \& Technology Task Book [revista en internet]. 2005 [consulta: 27/07/2014]. Disponible en: http://taskbook.nasaprs.com/ Publication/index.cfm?action=public_query_taskbook_ content\&TASKID=3790

23. Perchonok M, Antonini D. Thermostabilized shelf life study. Proceedings from the NASA Human Research Program Investigators Workshop [revista en internet]. 2008 [consulta: 27/07/2014]. Disponible en: http://ntrs.nasa.gov/archive/ nasa/casi.ntrs.nasa.gov/20080010623.pdf

24. Fu B, Nelson PE, Irvine R, Kanach LL. Processing of nutritious, safe and acceptable foods from CELSS candidate crops. Adv Space Res. 1996; 18(1-2): 241-50.
25. Smith SM, Zwart SR, Block G, Rice BL, Davis-Street JE. The nutritional status of astronauts is altered after long-term space flight aboard the International Space Station. J Nutr. 2005; 135(3): 437-43.

26. Zwart SR, Kloeris VL, Perchonok MH, Smith M. Assessment of nutrient stability in foods from the space food system after long-duration spaceflight on the ISS. J Food Sci. 2009; 74(7): H209-17.

27. Bowman BA, Russell RM. Present knowledge in nutrition. Washington, DC: ILSI Press; 2001. p. 805. 